\title{
Multi-pass resonator design for energy scaling of mode-locked thin-disk lasers
}

\author{
K. Schuhmann* ${ }^{\mathrm{a}, \mathrm{b}}, \mathrm{K} . \mathrm{Kirch}^{\mathrm{a}, \mathrm{b}}$, A. Antognini ${ }^{\mathrm{a}, \mathrm{b}}$ \\ ${ }^{a}$ Institute for Particle Physics, ETH, 8093 Zurich, Switzerland \\ ${ }^{\mathrm{b}}$ Paul Scherrer Institute, 5232 Villigen-PSI, Switzerland
}

\begin{abstract}
We present a novel multi-pass resonator architecture paving the way for pulse energy scaling of mode-locked thin-disk lasers. It consists of a concatenation of nearly identical optical segments, each segment corresponding to a round-trip in an optically stable cavity containing an active medium exhibiting soft aperture effects. Contrarily to state-of-the-art multi-pass oscillators based on imaging schemes, the stability region (for variations of the active medium thermal lens) of the here disclosed resonator architecture does not shrink with the number of passes. As a result, the proposed architecture enables the realization of an arbitrarily large number of passes at the thin-disk at a given pump power without the reduction of output power seen in the imaging-based state-of-the-art multi-pass oscillators.
\end{abstract}

Keywords: Thin-disk lasers, Laser resonators, Mode-locked lasers, Thermal lens effect, Ultrafast lasers, Energy scaling, Soft aperture

\section{MOTIVATION}

Ultra-short laser pulse sources [1], [2] enable a large variety of fundamental physics investigations, as well as technological and industrial applications. Many applications in industry and strong-field physics will tremendously benefit from the increase of the pulse energy in the $\mathrm{mJ}$ regime at few $\mathrm{MHz}$ repetition rates [3]: on one hand production throughput and material compendium extension especially for materials where non-linear multi-photon absorption is required, and on the other hand, reduced measurement times, increased signal to noise, and new scientific possibilities. Mode-locked thin-disk lasers [4], [5] are widely used in research laboratories and in industry because of their power scaling and high pulse energy capabilities [6], [7], [8], [9], [10], [11].

The output pulse energy $\mathrm{E}$ of a mode-locked thin-disk laser can be increased, at a given average output power $\mathrm{P}_{\text {avg }}$, by reducing the laser repetition rate $\mathrm{f}_{\text {rep }}$, given the simple relation $\mathrm{E}=\mathrm{P}_{\text {avg }} / \mathrm{f}_{\text {rep }}$ from energy conservation. Smaller repetition rates can be achieved simply by increasing the oscillator cavity length. One successful way to increment the resonator length was obtained by inserting into the cavity a Herriott cell [12], [13]. However, the elevated intra-cavity pulse energy achieved in this way required operation of the oscillator in an evacuated environment to avoid detrimental non-linear effects in air [13].

The cavity length can be also increased by folding the laser beam at the active medium (thin-disk) several times per round-trip [6], [14]. The large gain per round-trip achievable with such an active multi-pass cell enables large output coupling, which brings along a reduction of the intra-cavity power. Hence, this scheme providing a long cavity and decreased intra-cavity intensity is twofold advantageous and qualifies this resonator architecture for industrial applications as it allows operation in air. Another important feature of a multi-pass resonator scheme is the reduction of Q-switching instabilities due to a linear decrease of the gain saturation fluence with the number of reflections at the thindisk [15], [16].

The multi-pass active cells realized to date [6], [14] are based on relay 4f-imaging: $4 \mathrm{f}$ optical segments are used to image the thin-disk from pass to pass at the active medium so that the beam propagation in the active multi-pass cell proceeds following the scheme disk-4f-disk-4f-disk- $4 \mathrm{f} . .$. . The $4 \mathrm{f}$ propagation from the optical point of view corresponds to an effective propagation of zero length and it does not provide stability for misalignment or variation of the thin-disk focal strength. Hence, to realize a stable laser operation, the $4 \mathrm{f}$ multi-pass cell has to be embedded in a stable optical resonator [6].

*skarsten@phys.ethz.ch; phone: +41446332013

Solid State Lasers XXVI: Technology and Devices, edited by W. Andrew Clarkson, Ramesh K. Shori, Proc. of SPIE Vol. 10082, 100820J · ( 2017 SPIE · CCC code: 0277-786X/17/\$18 · doi: 10.1117/12.2251913 
The $4 \mathrm{f}$ multi-pass cell with $\mathrm{N}$ number of passes can be described as a single-pass having a total optical length of $\mathrm{L}_{\text {multi-pass }}=(\mathrm{N}-1) \cdot \mathrm{L}$, a gain of $\mathrm{g}_{\text {multi-pass }}=\mathrm{g} \cdot \mathrm{N}$, and an active medium dioptric power of $\mathrm{V}_{\text {multi-pass }}=\mathrm{N} \cdot \mathrm{V}$, where $\mathrm{L}=4 \mathrm{f}$ represents the length of a single $4 \mathrm{f}$-imaging stage, $\mathrm{g}$ the single-pass gain, and $\mathrm{V}$ the thin-disk dioptric power. Due to these cumulative effects, the resonator stability region [17] of an oscillator containing such a multi-pass 4f-based cell shrink linearly with the number of passes $\mathrm{N}$ as demonstrated in Figure 1 [18]. This shrinking presently limits energy and power scaling [19] of state-of-the-art thin-disk mode-locked lasers.

Another power scaling limitation of thin-disk laser has been recently disclosed [20]. It is related to a self-driven growth of misalignment arising from variations of the thermal lens at the thin-disk caused by the excursion of the laser Eigenmode from the optical axis. The sensitivity of the $4 \mathrm{f}$-based multi-pass oscillators to this misalignment increases linearly with the number of passes $\mathrm{N}$. On the contrary, multi-pass resonators based on the here presented architecture can be designed to avoid this limitation [20].

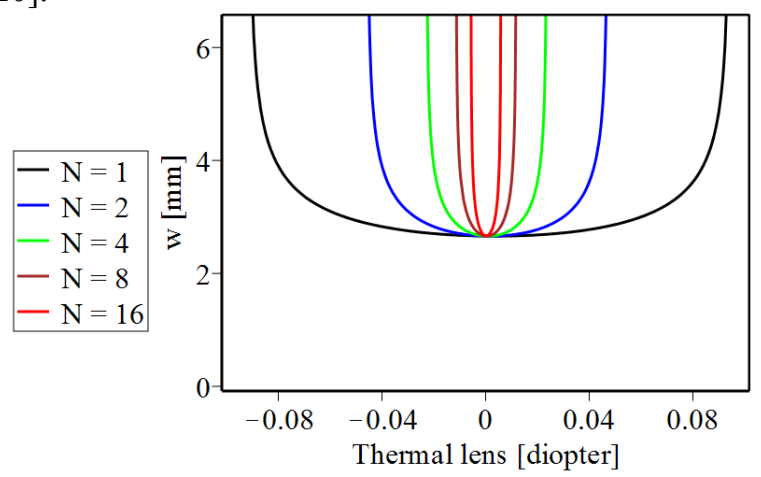

Figure 1: Stability plots of multi-pass resonators based on $4 \mathrm{f}$-imaging stages. Plotted are the cavity Eigen-mode (TEM00-mode) waist $\mathrm{w}$ at the thin-disk position for variations of the thin-disk thermal lens from the layout value. The shrinking of the stability region with the number of passes $\mathrm{N}$ per cavity round-trip arising in $4 \mathrm{f}$-based multi-pass resonators is demonstrated. These plots have been computed using the ABCD-matrix formalism and by embedding the $4 \mathrm{f}-$ based multipass cell at the thin-disk position of the stable resonator depicted in Figure 2. A wavelength of $1030 \mathrm{~nm}$ was assumed. Note however, that for a given Eigen-mode size the stability plots do not depend on the specific layout of the resonator.

In summary, the $4 \mathrm{f}$-based multi-pass oscillators show a limited energy scaling (capitalizing only on the advantages related to the long cavity length and the reduction of the intra cavity circulating intensity) but suffer for the shrinking of the stability region with the number of passes which reduces the maximal achievable output power. In this paper a novel multi-pass resonator scheme is presented which overcomes the thermal lens related power and energy limitations of state-of-the-art multi-pass mode-locked laser oscillators.

(a)
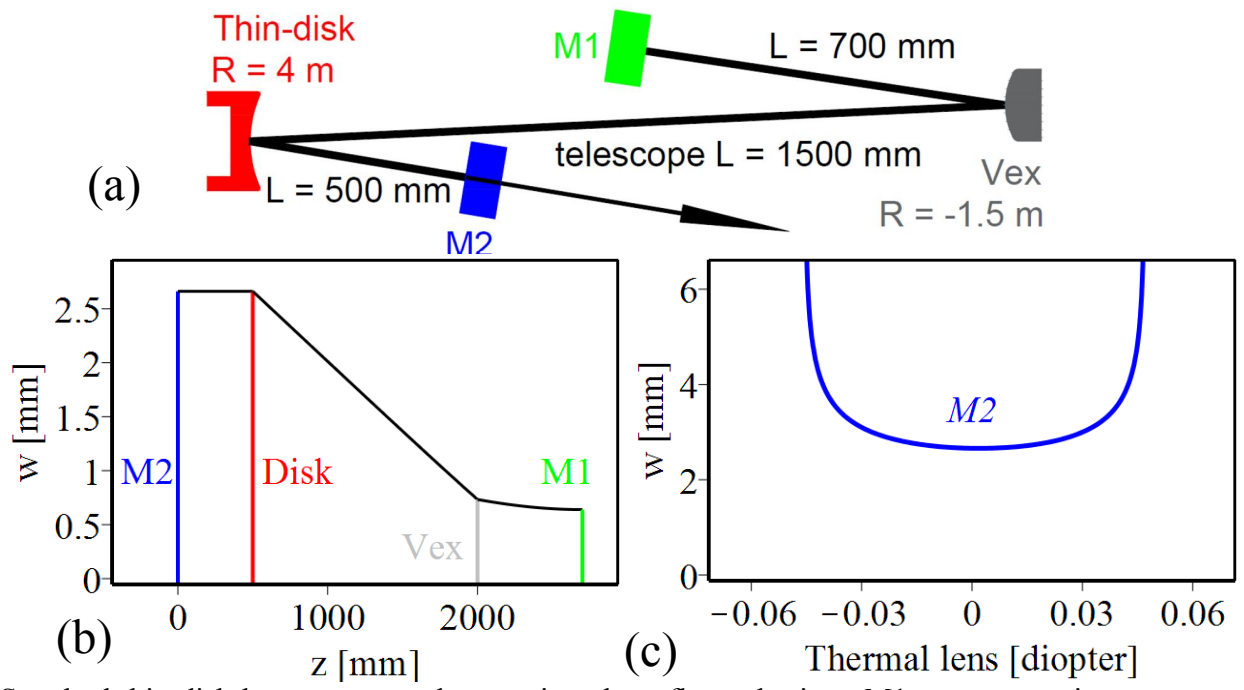

Figure 2: (a) Standard thin-disk laser resonator layout given by a flat end-mirror M1, a convex mirror, a concave thin-disk (red) and a flat end-mirror M2. (b) Corresponding Eigen-mode (TEM00-mode) waist w evolution along the resonator. The position of the optical elements is indicated by the vertical lines. (c) Corresponding stability plot. Plotted is the Eigen-mode waist at the M2 mirror for variations of the thin-disk thermal lens from the layout value. 


\section{NEW MULTI-PASS RESONATOR CONCEPT}

The multi-pass resonator we are proposing is based on a concatenation of identical (or nearly identical) segments. Each segment corresponds to a round-trip in an optically stable resonator containing one pass (or more) on the same active medium, which exhibits soft-aperture effects. The working principle and a possible realisation of this concatenation of segments can be deduced by comparing Figure 2 with Figure 3. Indeed, the multi-pass oscillator presented in Figure 3 (a) results from the concatenation of 8 identical segments given in Figure 2 (a). The resulting multi-pass resonator is inheriting the Eigen-mode properties of the underlying segment as can be seen by comparing Figure 2 (b) with Figure 3 (c). Therefore, the underlying segment has to be designed to be stable and insensitive to variations of thermal lens effects.

The stability region of the multi-pass resonator coincides with the stability region of a single segment provided all segments are identical. However, small differences between segments are unavoidable when practically realizing a multisegment oscillator as visible in Figure 3 (a) because of small variations of propagation lengths, incident angles and mirror curvatures.

Consideration of segment-to-segment asymmetries is essential for the understanding of the here proposed multi-pass resonator concept. In fact, these asymmetries prompt the formation of gaps in the stability region. Yet these gaps are suppressed by soft aperture effects naturally occurring in the pumped active medium as detailed in the next section.
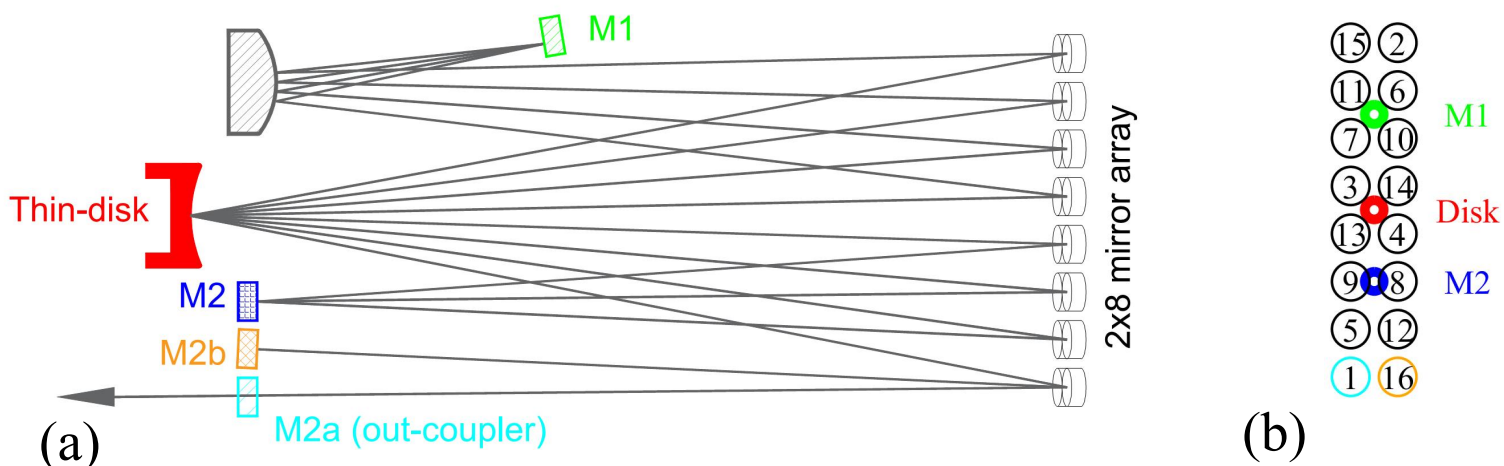

(a)

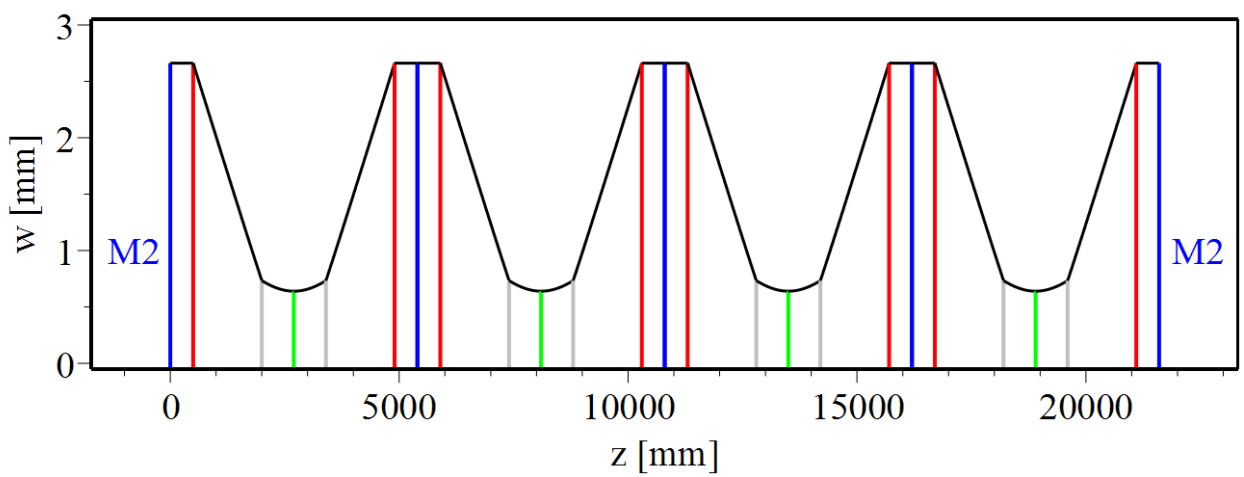

(c)

Figure 3: (a) Possible realization of a multi-pass oscillator with 16 reflections at the thin-disk per round-trip achieved by concatenating 8 identical segments as given in Figure 2. The beam routing can be realized with a mirror-array of 16 flat mirrors. One of the two end-mirrors M2a and M2b could be used as an out-coupler. (b) Working principle of the mirrorarray. The beam routing at the mirror-array plane follows the given numbering and is achieved by successive reflections at the thin-disk, mirror M1 and mirror(s) M2. The projection of these elements are indicated. (c) Eigen-mode (TEM00) waist w evolution in the multi-pass resonator of panel (a). The position of the optical elements is indicated by the vertical lines: In red are given the positions of the thin-disk, in blue the positions of the M2 mirrors, and in green the positions of the M1 mirror.

\section{THE IMPORTANCE OF SOFT APERTURE IN THE ACTIVE MEDIUM}

Even though thin-disk lasers are known for their small thermal lens, there are residual thermal lens effects which need to be taken into account. As detailed in [20] the thermal lens effects in the pumped thin-disk arise from the thin-disk-heat- 
sink bending, from the temperature dependence of the active medium refractive index, and the thermal expansion of the active medium in the laser direction. A simulation of the thermal lens effects expressed through the optical phase delay (OPD) experienced by a laser beam being reflected at the thin-disk is shown in Figure 4. In the region close to the thindisk axis $(\mathrm{x}=0)$ the OPD shows a quadratic profile. Therefore, in this region the thin-disk acts as a spherical lens. However, at the periphery of the pumped region, the OPD shows aspherical deformations which lead to additional cavity losses. These aspherical deformations, together with the absorption effects in the unpumped region, give rise to softaperture effects in the active medium.

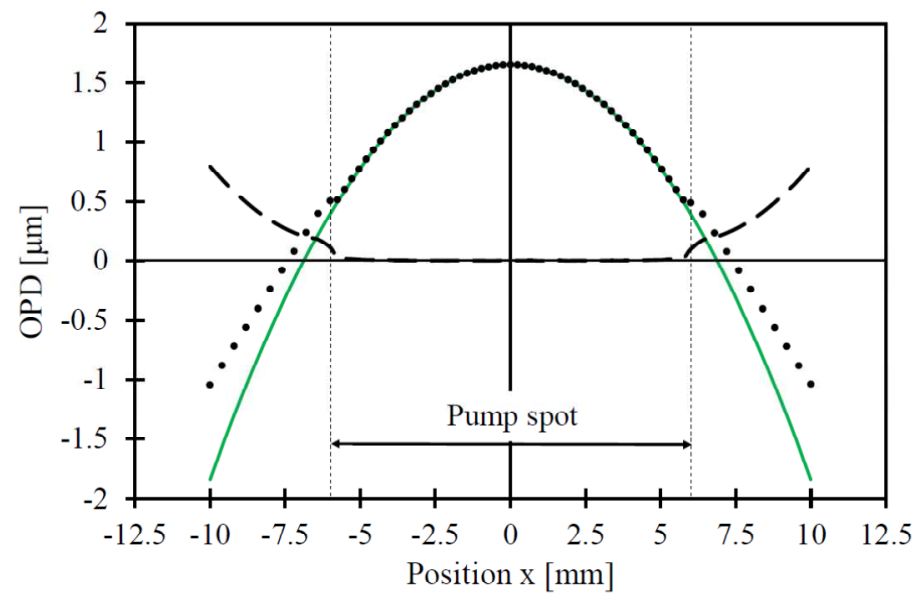

Figure 4: Numerical simulation of the thermal lens effect in a pumped thin-disk expressed using the space resolved optical phase delay (OPD) experienced by a laser beam being reflected at the thin-disk as detailed in [20]. The dots represent the total OPD computed using finite elements methods. The continuous green line represents the fit with a quadratic function of the total OPD, fitted only within the pumped region. The dashed line shows the difference between the total OPD and the fit displaying the aspherical deformations.

As anticipated previously, segment-to-segment asymmetries give rise to the formation of gaps within the stability region computed using identical segments (which corresponds to the stability region of a single segment resonator). The number of gaps increases with the number of segments of the multi-segment resonator while the size of these gaps increases with increasing segment-to-segment variations.
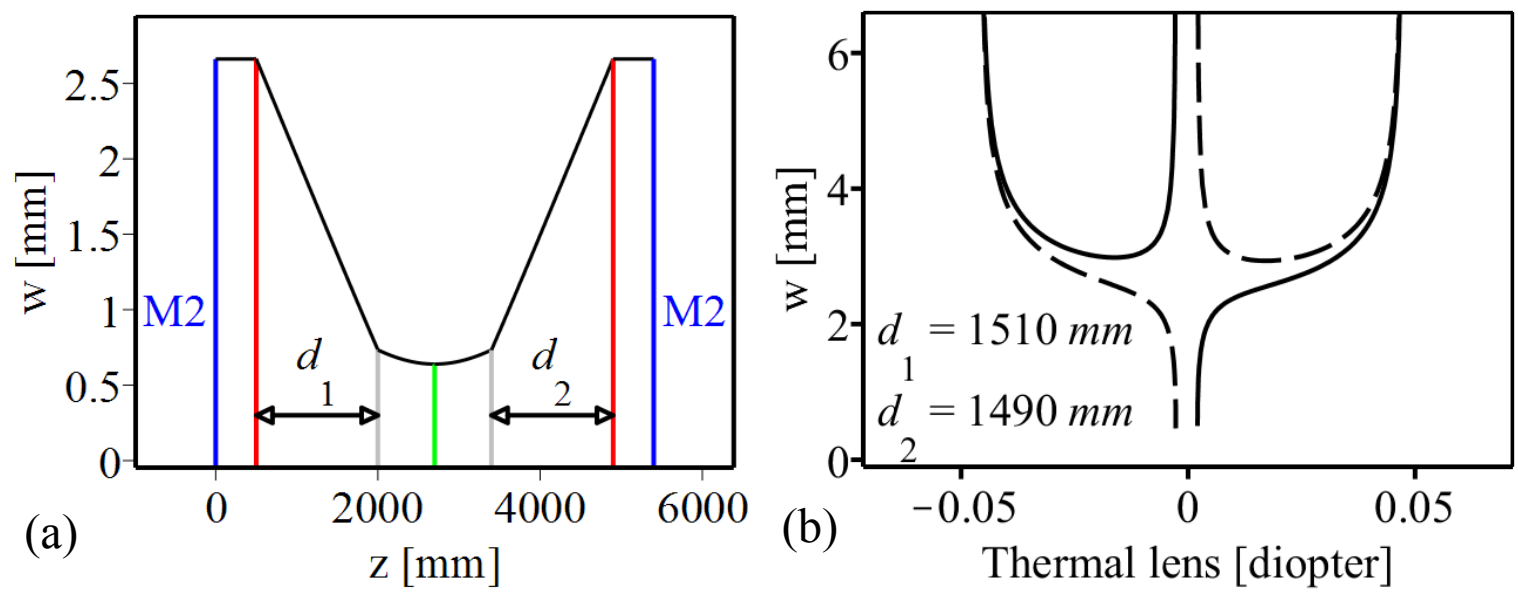

Figure 5: (a) Optical layout and Eigen-mode waist w evolution of a two-segment resonator with 4 reflections at the thin-disk per round-trip. The underlying segment is given in Figure 2. (b) Corresponding stability plot. Plotted is the Eigen-mode waist $\mathrm{w}$ at the two end-mirror positions M2 for variations of the thin-disk thermal lens from the layout value. A small asymmetry has been introduced $\left(\mathrm{d}_{1} \neq \mathrm{d}_{2}\right)$ between the two segments which induces a discontinuity (stability gap) in the center of the "original" stability region (cf. with Figure 2 (c)).

The formation of these gaps within the "original" (assuming identical segments) stability region as a consequence of small segment-to-segment differences is exemplified in Figure 5 for the particularly simple case that the multi-pass 
resonator is composed of only two segments. In the two-segment case, there is only one gap, and this gap arises in the center of the stability region. Similarly, for a multi-pass oscillator with several segments and various segment-to-segment deviations, a multitude of gaps would apparently fragment and reduce the "original" stability region.

It seems thus that segment-to-segment asymmetries would undermine the usefulness of the here proposed multi-pass resonator architecture. However, when soft aperture effects are accounted in the simulations, it turns out that the stability gaps within the "original" stability region disappear. This is demonstrated in Figure 6 for the same two-segment resonator of Figure 5. In fact, a comparison between Figure 6 (b) and Figure 5 (b) clearly reveals that the "original" stability region (compare with Figure 2 (c)) is restored if aperture effects are accounted for. The aperture effects reduce the infinitely large beam waist to small bumps so that the Eigen-mode properties of the multi-segment oscillator with small segment-to-segment variations are similar to the Eigen-mode properties of the multi-segment oscillator with identical segments which exactly correspond to the Eigen-mode properties of a single-segment oscillator.
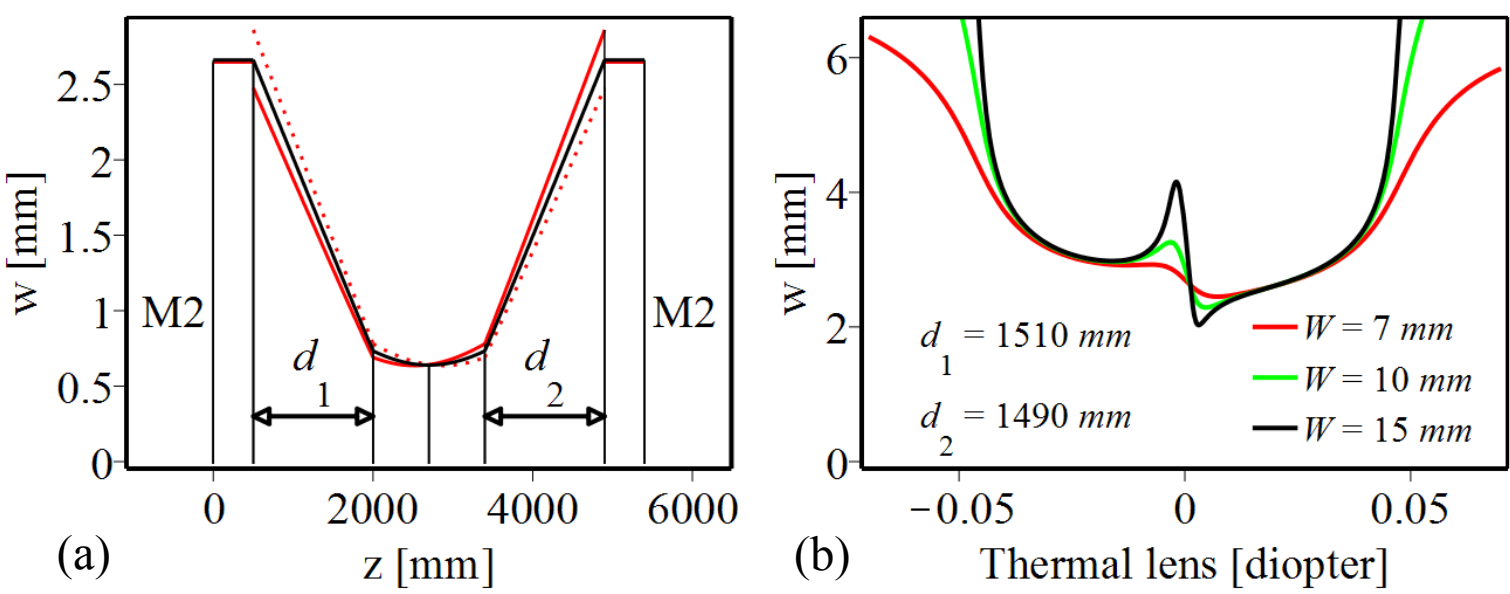

Figure 6: (a) Optical layout and Eigen-mode waist w of a two-segment resonator having small segment-to-segment asymmetries when accounting for aperture effects. The vertical lines represent the position of the optics as in Figure 5. The two red curves represent the Eigen-mode waist for the back (dotted) and forth (continuous) propagation when aperture effects are included. For comparison, the waist evolution without segment-to-segment asymmetries and aperture effects are given (black curve). (b) Corresponding stability plots. Plotted is the waist $\mathrm{w}$ at the left end-mirror position for variations of the thin-disk thermal lens for three Gaussian apertures with waist W.

The stability plots shown in the Figures 1, 2, 3 and 5 have been computed using the ABCD-matrix formalism. This formalism is a powerful instrument to compute Eigen-mode and stability regions of resonators. However, as already noted in [21], it is mostly used for computing bare resonators neglecting the effect of the transversely varying gain in the active material. Aperture effects which naturally occur in a pumped active medium mainly due to gain (absorption) in the pumped (unpumped) regions and related diffraction (see Figure 4) significantly affect the Eigen-mode and stability properties of the resonator [22]. These effects can be described approximatively by a Gaussian aperture at the active medium and included into the ABCD-matrix formalism as an imaginary lens [23], [24], [25], [26]. The ABCD-matrix describing the thin-disk can be thus written as

$$
M_{\text {thin-disk }}=\left[\begin{array}{cc}
1 & 0 \\
-\frac{1}{f}-i \frac{\lambda}{\pi W^{2}} & 1
\end{array}\right]
$$

where $\mathrm{W}$ represents the effective waist of the Gaussian aperture, $\lambda$ the laser wavelength and $\mathrm{f}$ the thin-disk focal length which also includes thermal lens effects.

Standard resonator designs do not include soft aperture effects because for a single-segment resonator the inclusion of aperture effects does not considerably alter the computed value of the Eigen-mode size for thin-disk thermal lens variations within the stability region (computed without aperture effects). For dioptric power outside the stability range (computed without aperture effects), the inclusion of aperture effects results in Eigen-modes with finite waist which implies an extension of the stability region [21], [27]. Therefore, the inclusion of aperture effects shows that in principle laser operation may also occur outside the "original" stability region. Yet this extension has no practical relevance because outside of the "original" stability range the round-trip losses caused by the aperture are increasing dramatically [17], [21]. 
Contrarily, aperture effects need to be included in the simulations of multi-segment resonators having small segment-tosegment deviations. Simulating multi-segment resonators with small segment-to-segment deviations without accounting for soft aperture effects produces wrong results because it predicts the formation of gaps within the stability region which does not occur in reality. The inclusion of these soft apertures into the simulations suppresses these gaps as illustrated in Figure 6 for the particular case of a two-segment resonator and leaves small residual fluctuations of the Eigen-mode waist. However, it is important to stress that the general behavior of the stability regions does not critically depend on the exact value of the assumed aperture waist $W$ as can be deduced by comparing the various curves of Figure 6 .
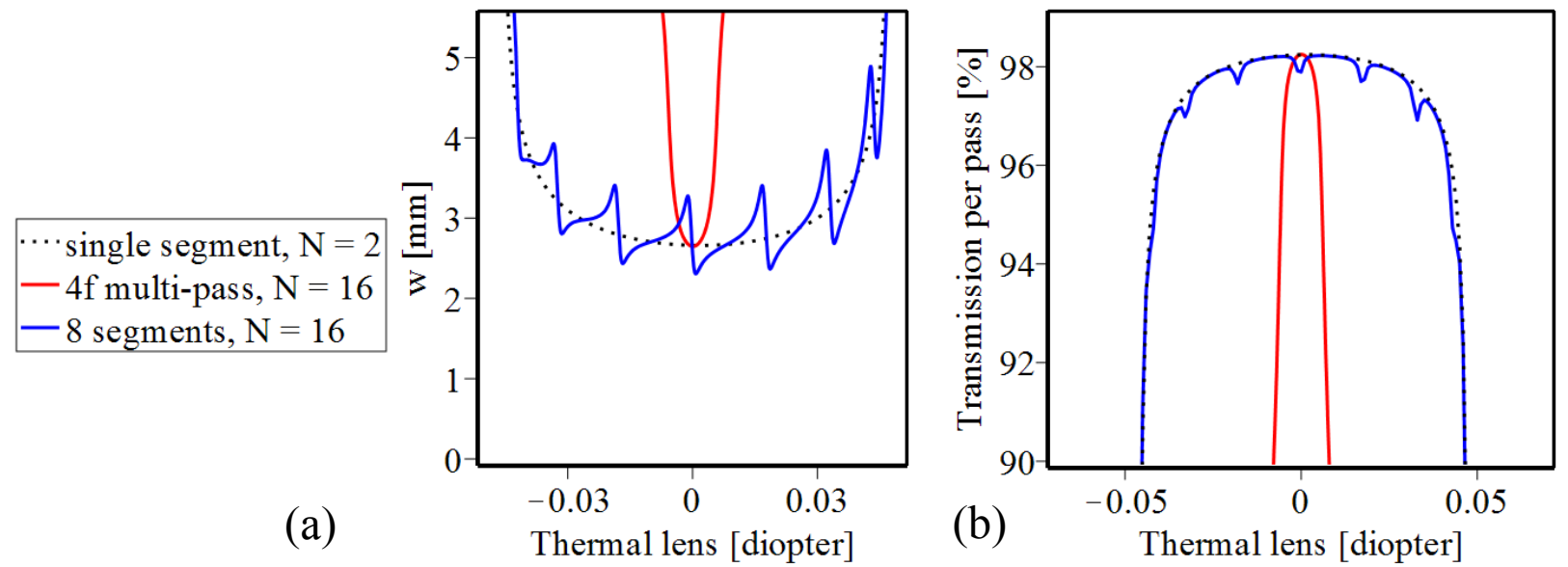

Figure 7: (a) Stability properties of three multi-pass resonator designs having same Eigen-mode waist w (in the center of the stability region), 16 reflections per round-trip at the active medium and a gain medium with a Gaussian aperture of $\mathrm{W}=20$ $\mathrm{mm}$. The black dotted curve represents the stability plot of the single-segment resonator of Figure 2. It serves as a reference. The blue continuous curve ( 8 segments, each containing two passes) represents the stability plot for a resonator based on our design as a succession of nearly-identical segments as given in Figure 3. The fluctuations arise from a small segment-tosegment asymmetry: the distance disk to convex mirror in the first segment has been assumed to be $1518 \mathrm{~mm}$ i.e., $20 \mathrm{~mm}$ longer than in the other segments. Besides these fluctuations, the stability plot of the multi-segment resonator is essentially identical to the stability plot of the single-segment resonator. The red curve represents the stability plot for a $4 \mathrm{f}$-based multipass resonator. Its stability region is 8 times smaller than the stability region of the single-segment resonator. (b) Corresponding average (over a round-trip) transmission through the Gaussian aperture at the thin-disk for variations of the thin-disk thermal lens.

This behavior can be generalized to resonators with several segments. Figure 7 shows that the stability region, (mode waist) and average losses per pass for the 8-segment multi-pass resonator of Figure 3 with small segment-to-segment deviations turn out to be practically identical with the stability region (mode waist) and losses per pass of the singlesegment resonator (compare the blue continuous and the black dotted lines of Figure 7). The same figure for comparison also shows the smaller stability range featured by the multi-pass resonator based on $4 \mathrm{f}$-imaging stages having the same number of passes $\mathrm{N}$ and beam waist $\mathrm{w}$ at the active medium.

In summary, the soft aperture effects occurring naturally in the pumped active medium grant the realization of a multipass oscillator as a concatenation of several nearly-identical optical segments. When considering aperture effects, the stability region and losses per pass of our multi-segment resonator is practically identical to the stability region of the single-segment resonator. Therefore, contrarily to the $4 \mathrm{f}$-based multi-pass resonator, the stability region of our multi-pass resonator does not shrink with the number of passes.

\section{PROOF OF PRINCIPLE}

For a proof of principle we transformed the multi-pass amplifier [28], [29] that we developed for spectroscopy of muonic atoms [30], [31] into a multi-pass oscillator by adding two end-mirrors. The multi-pass design schematically depicted in Figure 3 fulfills our requirements of sufficiently small segment-to-segment variations as it uses the same thin-disk and the same convex mirror in all segments. Moreover, the mirror array that is used to fold the beam providing several passes on the same thin-disk also guarantees similar path lengths and small incident angles. 


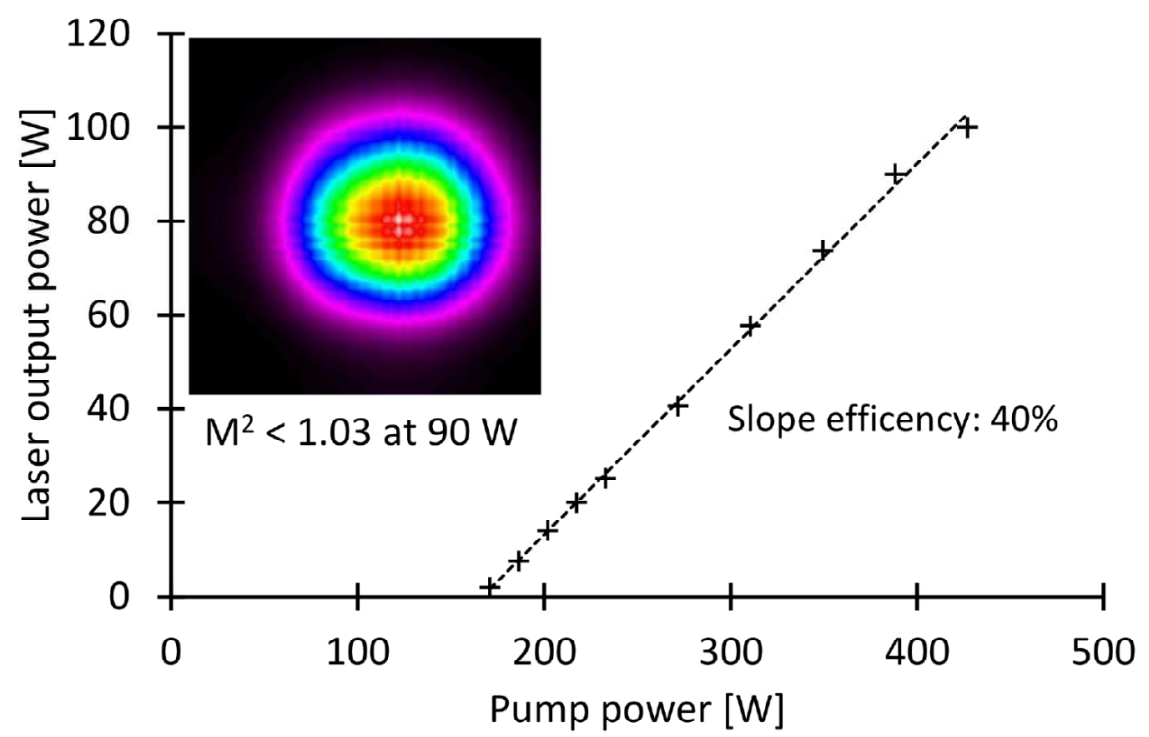

Figure 8: Input-output characteristics of the multi-pass resonator based on our design with 16 reflections per round-trip. The measurements have been accomplished in a cw operation for a Yb:YAG thin-disk of $345 \mathrm{~mm}$ thickness, a $940 \mathrm{~nm}$ pump wavelength and an out-coupling mirror reflectivity of $50 \%$. The inset shows the measured output beam.

The multi-pass oscillator (with 16 reflections at the thin-disk per round-trip) whose underlying segment specifications are given in Figure 2 (a) has been tested in cw mode using a flat out-coupler with $50 \%$ transmission. As active medium, a $345 \mathrm{~mm}$ thick $\mathrm{Yb}$ :YAG thin-disk with $5 \%$ nominal doping concentration contacted by TRUMPF to a water-cooled CVD-diamond heat sink has been used. Even though the choice of the thin-disk parameters were optimized for low repetition rate Q-switched operation, encouraging output powers and slope efficiency ( $40 \%$ in fundamental mode operation) have been observed as shown in Figure 8. The laser was designed with a beam waist of $\mathrm{w}=2.6 \mathrm{~mm}$ associated with a pumped area of $\mathrm{A}_{\text {pump }}=1.8 \mathrm{~cm}^{2}$. The measured threshold pump intensity was $\mathrm{I}_{\text {thresehold }}=370 \mathrm{~W} / \mathrm{cm}^{2}$ to be compared with the typical thin-disk limits of about $I_{\max }=8 \mathrm{~kW} / \mathrm{cm}^{2}$. This measurement represents the first preliminary demonstration of the applicability of our multi-pass oscillator concept, in particular showing that the soft aperture effects naturally present in the pumped active medium are sufficient to suppress the instabilities related to the various segment-to-segment asymmetries associated with the practical realization of a multi-pass scheme.

\section{CONCLUSIONS}

We have presented a multi-pass resonator architecture as a sequence of nearly-identical optically stable segments, each segment containing the same active medium featuring soft-aperture effects. The stability region of such a multi-segment resonator does not decrease with increasing number of segments provided segment-to-segment asymmetries are small.

This is achievable using the same active medium in all passes. Larger segment-to-segment deviations would cause increased losses (decreased transmission through the aperture) which strongly reduce laser efficiency or even disrupt laser operation.

We have demonstrated that it is essential to include soft aperture effects occurring in the active medium into the simulations. They suppress the formation of gaps within the stability region which would arise as a consequence of small segment-to-segment asymmetries associated with the practical realization of a multi-pass system. These aperture effects have been integrated into the ABCD-matrix formalism as lenses with imaginary focal length.

The here proposed multi-pass resonator layout is particularly suited for ultrafast lasers where the mode-locking mechanism is based on SESAM technologies [9]. The SESAM could be placed at the position of one of the resonator end-mirrors (e.g. mirror M2b in Figure 3) as at this position there is minimal intra-cavity intensity and the SESAM mirror would be intersected only once per round-trip.

This architecture solves the limitations of state-of-the-art multi-pass resonators based on $4 \mathrm{f}$-imaging stages which feature a shrinking of the stability region with the number of passes at the active medium. In addition, it also allows to avoid the power limitation related with the dynamical misalignment instability reported in [20]. Power scaling of mode-locked lasers in the $10 \mathrm{~kW}$ regime is thus conceivable using the here presented multi-pass resonator architecture and the newly developed SESAM technology [32]. 


\section{ACKNOWLEDGEMENT}

We acknowledge the support from the Swiss National Science Foundation Project SNF 200021_165854, the ETH Femtosecond and Attosecond Science and Technology (ETH-FAST) initiative as part of the NCCR MUST program and the ERC CoG. \#725039.

\section{REFERENCES}

[1] U. Keller, "Ultrafast solid-state laser oscillators: a success story for the last 20 years with no end in sight," Applied Physics B, vol. 100, pp. 15-28, 2010.

[2] F. Krausz and M. Ivanov, "Attosecond physics," Rev. Mod. Phys. 81, vol. 81, pp. 163-234, 2009.

[3] T. Sudmeyer, S. V. Marchese, S. Hashimoto, C. R. E. Baer, G. Gingras, B. Witzel and U. Keller, "Femtosecond laser oscillators for high-field science," Nature Photon, vol. 2, pp. 599-604, 2008.

[4] A. Giesen, H. Hügel, A. Voss, K. Wittig, U. Brauch and H. Opower, "Scalable concept for diode-pumped highpower solid-state lasers," Applied Physics B, vol. 58, pp. 365-372, 1994.

[5] A. Giesen, J. Speiser, R. Peters, C. Kränkel and K. Petermann, "Thin-Disk Lasers Come of Age," Photonics Spectra, 2007.

[6] T. Gottwald, C. Stolzenburg, D. Bauer, J. Kleinbauer, V. Kuhn, T. Metzger, S. Schad, D. Sutter and A. Killi, "Recent disk laser development at trumpf," in Proc. SPIE 8898, San Francisco, 2012.

[7] J. Mende, E. Schmid, J. Speiser, G. Spindler and A. Giesen, "Thin disk laser: power scaling to the kW regime in fundamental mode operation," in Proc. SPIE 7193 Solid State Lasers XVIII: Technology and Devices, San Francisco, 2009.

[8] M. Larionov and J. Neuhaus, "Regenerative thin disk amplifier with a pulse energy of $120 \mathrm{~mJ}$ at $1 \mathrm{kHz}$," in Advanced Solid State Lasers, Shanghai China, 2014.

[9] C. Saraceno, F. Emaury, C. Schriber, A. Diebold, M. Golling, T. Sudmeyer and U. Keller, "Towards oscillator driven strong-field experiments using," in International Conference on Ultrafast Phenomena, Okinawa Japan, 2014.

[10] J.-P. Negel, A. Voss, M. A. Ahmed, D. Bauer, D. Sutter, A. Killi and T. Graf, "1.1 kw average output power from a thin-disk multipass amplifier for ultrashort laser pulses," Opt. Lett., vol. 38, pp. 5442-5445, 2013.

[11] O. Pronin, J. Brons, C. Grasse, V. Pervak, G. Boehm, M.-C. Amann, A. Apolonski, V. L. Kalashnikov and F. Krausz, "High-power Kerr-lens mode-locked Yb:YAG thin-disk oscillator in the positive dispersion regime," Opt. Lett., vol. 37, pp. 3543-3545, 2012.

[12] M. Richardson and A. Zoubir, "High intensity MHz mode-locked". USA Patent Patent US 7590156 B1, 2009.

[13] C. Saraceno, F. Emaury, C. Schriber, M. Hoffmann, M. Golling, T. Südmeyer and U. Keller, "Ultrafast thin-disk laser with $80 \mu \mathrm{J}$ pulse energy and $242 \mathrm{~W}$ of average power," Optics Letters, vol. 39, no. 1, pp. 9-12, 2014.

[14] D. Bauer, I. Zawischa, D. H. Sutter, A. Killi and T. Dekorsy, "Mode-locked yb:yag thin-disk oscillator with $41 \mu \mathrm{j}$ pulse energy at $145 \mathrm{w}$ average infrared power and high power frequency conversion," Opt. Express, vol. 20, pp. 9698-9704, 2012.

[15] C. Hönninger, R. Paschotta, F. Morier-Genoud, M. Moser and U. Keller, "Q-switching stability limits of continuous-wave passive mode locking," J. Opt. Soc. Am. B , p. 46-56, 1999.

[16] B. Dannecker, X. Délen, K. S. Wentsch, B. Weichelt, C. Hönninger, A. Voss, M. A. Ahmed and T. Graf, "Passively mode-locked Yb:CaF2 thin-disk laser," Opt. Express, vol. 22, pp. 22278-22284, 2014.

[17] V. Magni, "Resonators for solid-state lasers with large-volume fundamental mode and high alignment stability," Appl. Opt. 25, vol. 25, pp. 107-117, 1986.

[18] J. Speiser, "Thin disk laser-energy scaling," Laser Physics, vol. 19, pp. 274-280, 2009.

[19] C. R. E. Baer, O. H. Heckl, C. J. Saraceno, C. Schriber, C. Kränkel, T. Südmeyer and U. Keller, "Frontiers in passively mode-locked high-power thin disk laser oscillators," Opt. Express, pp. 7054-7065, 1332012. 
[20] K. Schuhmann, K. Kirch, F. Nez, R. Pohl and A. Antognini, "Thin-disk laser scaling limit due to thermal-lens," Appl. Opt., vol. 55, pp. 9022-9032, 2016.

[21] S. Gatz and J. Herrman, "Geometrical threshold zones and Gaussian modes in lasers with radially varying gain," Opt. Let., vol. 19, pp. 1696-1698, 1994.

[22] L. W. Casperon and A. Yariv, "The Gaussian Mode in Optical Resonators with a Radial Gain Profile," Appl. Phys. Lett., vol. 12, pp. 355-357, 1968.

[23] L. W. Casperon and S. D. Lunnam, "Gaussian Modes in High Loss Laser Resonators," Appl. Opt., vol. 14, pp. 1193-1199, 1975.

[24] J. Herrman, "Theory of Kerr-lens mode locking: role of self-focusing and radially varying gain," J. Opt.Soc. Am. B, vol. 11, pp. 498-512, 1994.

[25] H. Kogelnik, "On the Propagation of Gaussian Beams of Light Through Lenslike Media Inducing those with a Loss or Gain Variation," Appl. Opt., vol. 4, pp. 1562-1569, 1965.

[26] A. Siegman, Lasers, University Science Books, 1986.

[27] D. V. Willetts and M. R. Harris, "Output Characteristics of a Compact 1 J Carbon Dioxide Laser with a Gaussian Reflectivity Resonator," IEEE Journal of quantum electronics, vol. 24, pp. 849-855, 1988.

[28] A. Antognini, K. Schuhmann, F. D. Amaro and e. all, "Thin-Disk Yb:YAG Oscillator-Amplifier Laser, ASE, and Effective Yb:YAG Lifetime," Journal of Quantum Electronics, IEEE, vol. 45, no. 8, pp. 993-1005, 2009.

[29] K. Schuhmann, M. A. Ahmed, A. S. Antognini, T. Graf, T. W. Hänsch, K. Kirch, F. Kottmann, R. Pohl, D. Taqqu, A. Voss and B. Weichelt, "Thin-disk laser multi-pass amplifier," in Proceedings of SPIE, San Francisco, 2015.

[30] R. Pohl, A. Antognini, F. Nez, F. Amaro, F. Biraben, J. Cardoso, D. Covita, A. Dax, S. Dhawan, L. Fernandes, A. Giesen, T. Graf, T. Hänsch, P. Indelicato, L. Julien and C. Kao, "The size of the proton," Nature, vol. 466, pp. 213216, 08072010.

[31] A. Antognini, F. Nez, K. Schuhmann, F. D. Amaro, F. Biraben, J. M. R. Cardoso, D. S. Covita, A. Dax, S. Dhawan, M. Diepold, L. M. P. Fernandes, A. Giesen, A. L. Gouvea, T. Graf, T. W. Hnsch, P. Indelicato, L. Julien, C.-Y. Kao, P. Knowles, F. Kottmann and E., "Proton structure from the measurement of 2s-2p transition frequencies of muonic hydrogen," Science, vol. 339, pp. 417-420, 2013.

[32] A. Diebold, T. Zengerle, C. G. E. Alfieri, C. Schriber, F. Emaury, M. Mangold, M. Hoffmann, C. J. Saraceno, M. Golling, D. Follman, G. D. Cole, M. Aspelmeyer, T. Südmeyer and U. Keller, "Optimized SESAMs for kilowattlevel ultrafast lasers," Opt. Express, vol. 24, pp. 10512-10526, 2016. 